\title{
Superconducting current in narrow proximity wires
}

\author{
Frank K. Wilhelm, Andrei D. Zaikin, and Gerd Schön
}

Institut für theoretische Festkörperphysik, Universität Karlsruhe (TH), D-76128 Karlsruhe, Germany

The critical supercurrent in proximty-wires shows a surprising temperature dependence in the experiment [1]. This observation has lead to speculation whether there are new effects involved and/or the usual criterion for the dirty limit is not valid for this type of systems. We propose a simple SNS-model for this system and show analytically, that this unexpected scaling is already contained in a conventional description of this model by quasiclassical Green's functions in the dirty limit. Based n further numerical calculations, excellent agreement with the experimental results is obtained.

\section{Introduction}

Recent experiments [1] have shown, that a thin normal diffusive wire upon which an array of superconducting stripes is laid can carry a supercurrent. This is due to the well-known proximity effect [2]. As the $\mathrm{S}-\mathrm{N}$ contacts are very good, the penetration

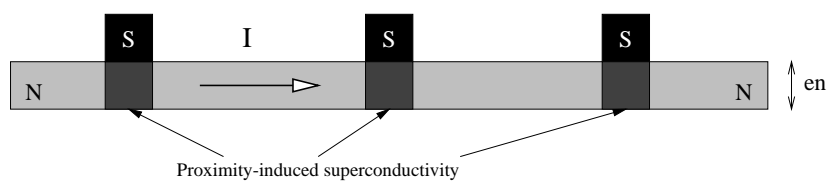

Figure 1: Experimental setup

depth of the pair amplitude into the normal wire $\xi_{N}=\sqrt{\frac{D}{2 \pi T}}$ is much bigger than the wire thickness $e_{N}$ at all experimental temperatures. This implies, that the pair amplitude in the regions covered by the stripes is identical to the bulk value and can be taken as constant over the cross section of the wire. Therefore, this structure is equivalent to a simple chain of SNS-junctions, so the supercurrent is a Josephson current [3]. However, comparison to standard results for such structures [2] failed [1]. The observed critical current $I_{c}(T)$ resembled to a clean $\left(I_{c}(T) \propto e^{-\frac{T}{T_{0}}}\right)$ rather than a dirty system. This has raised the question, whether the usual criterion between clean and dirty limit is correct for weak superconductivity or whether this effect has to be attributed to new quantum effects not contained in quasiclassical theory of superconductivity. However, we will demonstrate that neither of this is true, that the deviations are due to the use of Ginzburg/Landau theory in [2], which is not valid for the experimental range of tem- peratures and that a full quasiclassical calculation can explain the experiment quantitavely.

\section{Results}

Obviously, the critical current is determined by the longest SNS-cell serving as a "bottleneck", so it is sufficient to study a single cell.

\subsection{Analytical approximations}

However, in the case $d \gg \xi_{N}$, where $d$ is the distance of two superconducting banks, the mutual influence between the superconductors can be neglected and the solution can be decomposed into contributions from both sides, carrying a fixed phase. From this approximation, we get for the current

$$
\begin{aligned}
I & =\frac{64 \pi T}{e R_{N}} \sum_{\omega_{m}} \frac{d}{\xi_{N, \omega}} w(\omega, \Delta) e^{-\frac{d}{\xi_{N, \omega}}} \sin \phi(1) \\
w(\omega, \Delta) & =\frac{\Delta^{2}}{\left(\omega+\Omega+\sqrt{2\left(\Omega^{2}+\omega \Omega\right)}\right)^{2}}
\end{aligned}
$$

where $\phi$ is the phase difference between the superconductors, $R_{N}$ the Drude resistance and $\omega_{n}=$ $(2 n+1) \pi T \quad n=0,1,2 \ldots$ are fermion Matsubara frequencies. Furthermore, $\xi_{N, \omega}=\sqrt{\frac{D}{2 \omega}}, \Delta$ is the superconducting order parameter and $\Omega=\sqrt{\omega^{2}+\Delta^{2}}$. For $T \gg \epsilon_{d}=D / d^{2}$, all frequencies higher than the lowest one can be neglected. In the case $T \ll \Delta$, this gives the critical current by setting $\phi=\pi / 2$ :

$$
I_{c}=\frac{64 \pi}{3+2 \sqrt{2}} \frac{T}{e R_{N}} \frac{d}{\xi_{N}} \exp \left(-\frac{d}{\xi_{N}}\right)
$$

This result agrees with [4] and has the usual dirty limit form $I_{c} \propto T^{q} \exp \left(-\sqrt{\frac{T}{T_{0}}}\right)$ with $T_{0}=\frac{D}{2 \pi d^{2}}$ 

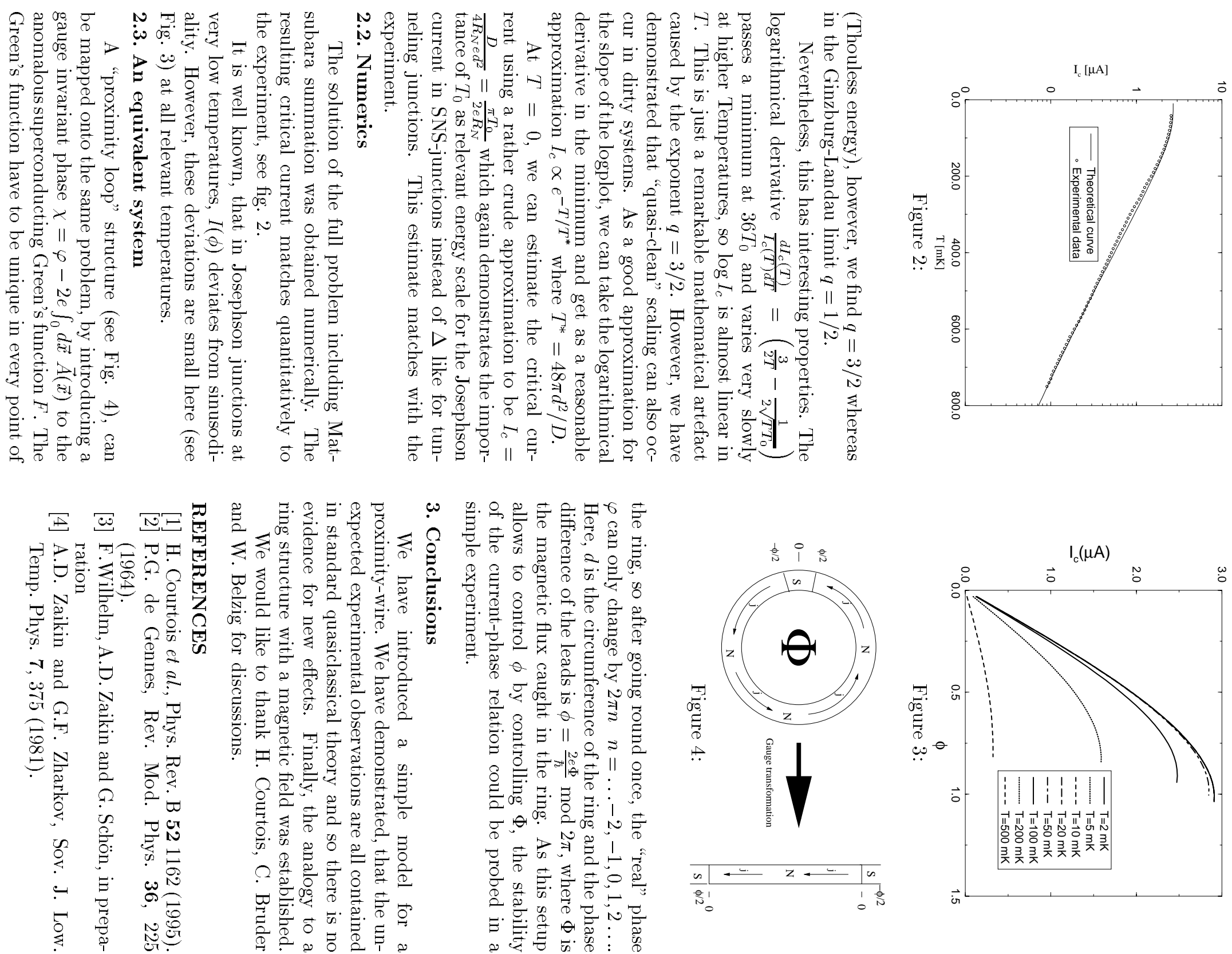